\title{
Empirical Approaches for Assessing Key Factors Pertaining to Greenhouse Gas Emissions in Early Stages of Projects
}

\author{
WOOSIK JANG ${ }^{\mathrm{a},}$, , SEOUNG BEOM NA ${ }^{\mathrm{b}}$, HARRY LEE \\ ${ }^{a}$ Department of Civil Engineering, Chosun University, Gwangju, Korea (Corresponding Author) \\ ${ }^{b}$ Department of Civil Engineering, Chosun University, Gwangju, Korea \\ ${ }^{c}$ Department of Civil Engineering, Chosun University, Gwangju, Korea
}

\begin{abstract}
Since the middle of the 18th century, the entire world has been devoted to industrial development. Based on the development of industry, many countries around the world have achieved rapid economic growth [1]. This resulted not only in economic growth but also a large increase in the amount of greenhouse gases (GHG), which is evident as climate change and environmental disruption. To overcome such adverse effects, this study focuses on assessing the key environmental factors at the early stage of a project for decision makers. To support this objective, the authors have collected 210 real road construction cases and investigated 23 dependent factors including design parameters and physical environment of construction projects. Subsequently, statistical analysis as factor selection and regression modeling is conducted to extract the key factors. As a result, a total of five factors are extracted: "Area of Rice field," "Total Project Cost," "Number of Bridges," "Design (maximum) Speed," and "Length of Earthwork" via regression analysis. Moreover, the proposed regression model accounts for $69 \%$ and $67 \%$ of the total variance of "Total Environmental Loads" indicated by $\mathrm{R}^{2}$ and adjusted $\mathrm{R}^{2}$, respectively, at $99 \%$ of the significance level. Thus, the proposed model is expected to play a positive role in the decision-making process based on mathematical and empirical evidences.
\end{abstract}

(C) 2019 The Authors. Published by Budapest University of Technology and Economics \& Diamond Congress Ltd.

Peer-review under responsibility of the scientific committee of the Creative Construction Conference 2019.

Keywords: Greenhouse gases (GHG); Environmental Variables; Decision Making; Regression model; Planning Phase; Construction Projects

\section{INTRODUCTION}

Since the middle of the 18th century, the world has been devoted to industrial development resulting in a substantially convenient and abundant life. As a result, many countries in the world are now urbanized, and more than half of the world's population lives in urban areas [2]. Economic growth is based on the emission of greenhouse gases (GHG) as a result of resource consumption. Thus, rapid economic growth and urbanization are not only the cause of serious pollution and exhaustion of resources, but also a primary factor in the greenhouse effect [1]. National Oceanic and Atmospheric Administration (NOAA) analyzed that the average temperature of the Earth's surface is constantly rising, and faster changes are observed as modernization accelerates [6]. This means that GHG emissions have exceeded the natural purification rate of the earth. Because of climate change, GHG can result in several diseases and damages to human kind. Moreover, climate change gives rise to abnormal conditions which poses threat to our lives. Fortunately, 
the concept of environmental protection is widely spreading to make the earth considerably sustainable. Following this trend, the Kyoto protocol was implemented in 1997 to reduce GHG emissions.

Nevertheless, sustainable growth is faced with problems such as egoism or chauvinism. The agreement was declined in the 17th Conference of Parties (COP 17) at the United Nations Framework Convention on Climate Change (UNFCCC) [7]. COP 17 aimed at new strategies for climate change, but only the renewal of the Kyoto protocol was agreed to. However, some of top emission countries decided and announced that the reject of new Kyoto protocol. Thus, the agreement of COP 17 is flawed in terms of substantial impact. Environment is not a subject of agreements and deals. Thus, new constraint for climate change should be specified at the earliest, and the preparation for sustainable growth is crucial. To control and manage sustainability in case of construction, the assessment of key impact factors is critical. Past research only dealt with cost or performance as the influencing factors. Further, these studies did not consider the aspect of sustainability, and so, their direct application to environmental factors is irrational. Consequently, assessing the key environmental impact factors in construction projects is important.

\section{EXPERIMENT}

The primary objective of this article is to identify the key factors that impact the environment and compare the results between the key factors of cost impacts in construction projects. In particular, the focus is on a road construction project in various landforms including mountains and river areas. The specific objectives of this study include; (1) examining the relationship between Total Environmental Loads (TEL) including bridging and tunneling works; (2) preliminary assessment of both key factors based on 210 road construction cases; and (3) comparison between both key factors and subsequent analysis of the results. Figure 1 shows the experimental procedure.
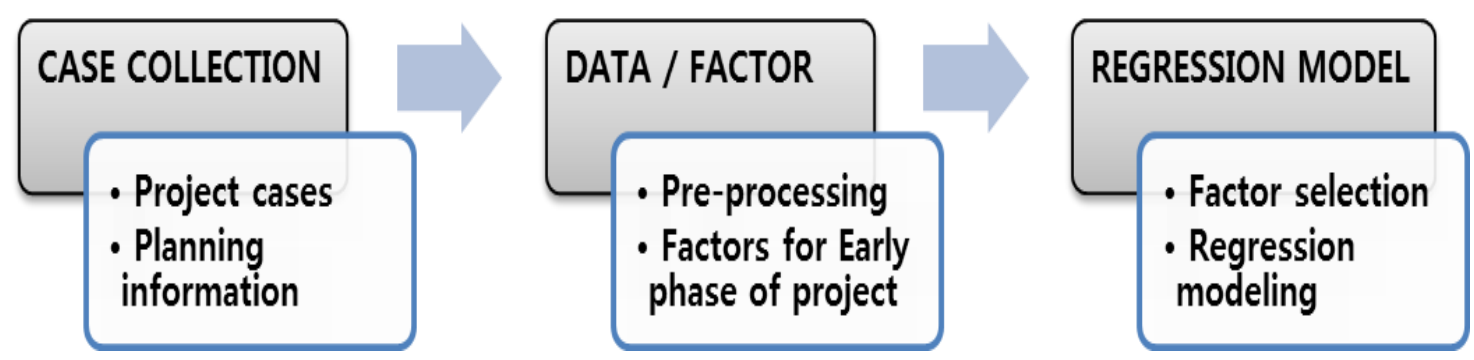

Fig 1. Experimental procedure

\subsection{Case Collection}

To ensure the reliability of the experiment, the authors collected several cases of road construction projects. Consequently, data for a total of 210 project cases were collected, which include the detailed information of the projects. Table 1 presents the nature of the collected cases. First, several projects are located in mountainous areas; mountainous areas are a regional characteristic of Korea, with more than $70 \%$ of the territory being in mountainous areas. Further, a few of the projects are located in the downtown area, which is a characteristic of the expressway that connects provinces or cities.

Table 1. Nature of collected project

\begin{tabular}{|c|c|c|}
\hline Category & Section & Rate $(\%)$ \\
\hline \multirow{3}{*}{ Site } & Downtown & 3.8 \\
\hline & Mountain & 62.6 \\
\hline & Plain & 33.6 \\
\hline \multirow{3}{*}{ Length } & Under $5 \mathrm{~km}$ & 20.9 \\
\hline & $5-10 \mathrm{~km}$ & 49.9 \\
\hline & Over $10 \mathrm{~km}$ & 32.2 \\
\hline \multirow{3}{*}{ Contract sum } & Under 50 billion & 30.3 \\
\hline & $50-100$ billion & 40.3 \\
\hline & Over 100 billion & 29.4 \\
\hline \multirow[b]{2}{*}{ Owner } & Public & 90 \\
\hline & $\begin{array}{c}\text { Private } \\
\text { (including PPP) }\end{array}$ & 10 \\
\hline
\end{tabular}


Woosik Jang, Seung Bum Na, Harry Lee/ Proceedings of the Creative Construction Conference (2019) 094 https://doi.org/10.3311/CCC2019-094

\subsection{Regression Model}

This study aims at developing a prediction model for decision makers. Further, it aims to support the conceptual planning phase of projects. Thus, the authors investigate the factors focusing on the collectable data in the early phase of a project from the collected projects including the bill of quantity (BOQ) and proposal report of each project. Using this process, a total of 23 factors that can represent the nature of the construction projects at the early phase were identified. Table 2 lists the details of these factors. Subsequently, the authors obtained the key impact factors using factor analysis and set up the regression model using SPSS program.

Table 2. Descriptions of factors

\begin{tabular}{cccc}
\hline Codes & Description & Codes & Description \\
\hline F1 & Type of Construction & F13 & Site location_Plain \\
\hline F2 & Regional Characteristics & F14 & Farm area \\
\hline F3 & Number of lanes & F15 & Rice field area \\
\hline F4 & Width of lanes & F16 & Plot area \\
\hline F5 & Total length of Roads & F17 & Mountain area \\
\hline F6 & Length of Earthwork & F18 & Total Area \\
\hline F7 & Level of Roads & F19 & Number of Bridge \\
\hline F8 & Design (maximum) Speed & F20 & Total length of Bridge \\
\hline F9 & Radius of Horizontal Curve & F21 & Number of Tunnel \\
\hline F10 & Maximum Incline & F22 & Total length of Tunnel \\
\hline F11 & Site location_ Down town & F23 & Total Project Cost \\
\hline F12 & Site location_Mountain, & & \\
\hline & & &
\end{tabular}

In details, all factors were investigated from BOQ and proposal of projects that have various scales and dimensions. Generally, factors are categorized in two sections such as dummy and independent variables. Total 7 factors are categorized as dummy variables i.e., F1, F2, F3, F7, F11, F12, and F13. Other factors are categorized as independent variables. Nevertheless, a total 103 cases can satisfy this information among the total 210 cases. Thus, to reduce the bias from missing or incomplete data set, the authors used only 103 cases to set up the regression model. After factor selection, the authors performed a regression analysis. Consequently, five factors were extracted as F15, F23, F19, F8, and F6. Each of the factors is highly significant at the 99\% significance level. Table 3 shows the results of the regression analysis.

Table 3. Results of regression analysis

\begin{tabular}{ccccc}
\hline & Coefficient & SE & $\mathrm{t}$ & Sign. \\
\hline (Constant) & -66616 & 22984 & -2.89 & 0.005 \\
\hline Rice field area & 0.070 & 0.23 & 3.06 & 0.003 \\
\hline Total Project Cost & 0.000004 & .000 & 5.75 & 0.000 \\
\hline Number of Bridge & -6450 & 2189 & -2.94 & 0.004 \\
\hline Design (maximum) Speed & 1119 & 304 & 3.67 & 0.000 \\
\hline Length of Earthwork & 2.783 & 0.886 & 3.14 \\
\hline $\mathrm{N}$ & \multicolumn{3}{c}{0.002} \\
\hline $\mathrm{R}^{2}$ & 0.693 \\
\hline Adjusted $\mathrm{R}^{2}$ & 0.677 \\
\hline SE; Standard Error, $\mathrm{t}$; t-Statistics, Sign; Significance, N; Number of samples
\end{tabular}

\section{DISCUSSIONS AND CONCLUSIONS}

This paper deals with the assessment of key impact factors for construction project from an environmental perspective. Moreover, it also focused on the early phase of project (conceptual planning phase). To achieve these objectives, first, the authors collected 210 real project cases. Subsequently, they identified the related factors regarding TEL (Total 
Woosik Jang, Seung Bum Na, Harry Lee/ Proceedings of the Creative Construction Conference (2019) 094 https://doi.org/10.3311/CCC2019-094

Environmental Loads). After data processing, the authors applied SPSS program for variable selection and regression analysis. Through variable selection, correlated and overlapped factors were eliminated. Finally, regression model was suggested for TEL estimation. Statistically acceptable results are obtained via $\mathrm{R}^{2}(0.693)$ and adjusted $\mathrm{R}^{2}(0.677)$ considering that the previous researches suggested $30 \%$ to $50 \%$ of error rate at the early phase of a project [3] [4] [5]. However, this paper has certain limitations with regard to the use of simple statistical approach, and bias of data, which composed of Korean projects. However, it will be considered in future research. Despite these limitations, this paper analyses 210 real construction cases focusing on road project and satisfies the general range of expectations. Thus, it is expected to play a positive role in the decision-making process based on mathematical and empirical evidences.

\section{Acknowledgements}

This research was supported by a grant (18IFIP-C146568-01) from Plant Research and Development Program funded by Ministry of Land, Infrastructure and Transport of Korean government.

\section{References}

[1] Baeumler et al. (2012), Liu et al (2013), Yang et al (2017)

[2] Sun et al. (2016), Mat et al. (2016)

[3] Ashworth, A. \& Skitmore, M., Accuracy in estimating. Occasional Paper. London: Chartered Institute of Building (1982)

[4] Caltrans, Project Development Procedures Manual. Ch.20: Project Development Cost Estimates (2007) https://doi.org/ 10.1061/(ASCE)07339364(2007)133:1(91)

[5] Liu, L. \& Zhu, K., Improving cost estimates of construction projects using phased cost factors. Journal of Construction Engineering and Management, (2007), Vol. 133, pp. 91-95.

[6] National Oceanic and Atmospheric Administration (NOAA), Atmospheric CO2 at Mauna Loa-Hawaii, http://www.esrl.noaa.gov/gmd/ccgg/trends/\#mlo_data (2012)

[7] United Nations Framework Convention on Climate Change (UNFCCC), Test of the Kyoto Protocol, http://unfccc.int/key_documents/kyoto_protocol/items/6445.php (2012) 